\title{
Studies for the effect of the positive $Q$-value neutron transfers on sub-barrier heavy-ion fusion
}

\author{
H.M. Jia, C.J. Lin, L. Yang, X.X. Xu, N.R. Ma, L.J. Sun, \\ F. YANG, Z.D. Wu, H.Q. Zhang, Z.H. LiU and D.X. WANG
}

China Institute of Atomic Energy, P. O. Box 275(10), Beijing 102413, P. R. China

\begin{abstract}
This contribution reports the recent experimental studies for the coupling effect of the positive $Q$-value neutron transfer (PQNT) channels on near-barrier fusion with heavy-ions, measured by an electrostatic deflector setup. This effect is expected to be important in some situations. In this presentation, the experimental studies for the nearbarrier fusion of ${ }^{32} \mathrm{~S}+{ }^{90,94,96} \mathrm{Zr},{ }^{16} \mathrm{O}+{ }^{76} \mathrm{Ge},{ }^{18} \mathrm{O}+{ }^{74} \mathrm{Ge}$, and ${ }^{16,18} \mathrm{O}$ $+{ }^{58} \mathrm{Ni}$ are reported. Also a method, based on the CCFULL calculations with suitable inelastic couplings, to sort out the PQNT effect is presented.
\end{abstract}

\section{Introduction}

Near-barrier fusion with heavy-ions offers a good platform to study the two basic mechanisms of tunneling and coupling $[1,2]$ in the quantum world. The involvement of the couplings may lead to strong sub-barrier fusion enhancement in some situations. So far, couplings to these collective excitation states can be described well in near-barrier fusion by using the coupledchannels (CC) theory [2]. While couplings to nucleon transfers are even troublesome and have not yet been well understood. 


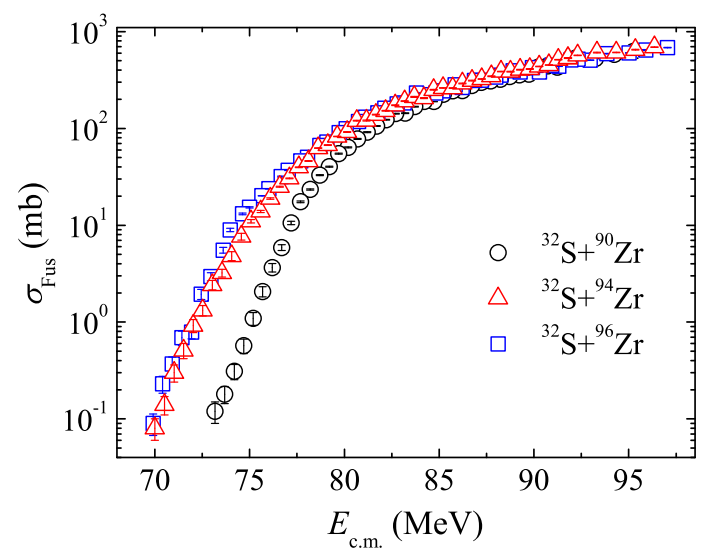

Figure 1: Experimental near-barrier fusion excitation functions of ${ }^{32} \mathrm{~S}+{ }^{90,94,96} \mathrm{Zr}$.

For the transfer reactions, neutron transfers might be more important than proton transfers especially at sub-barrier energy region. The possible effect of positive $Q$-value neutron transfers (PQNT) on near-barrier fusion was first discovered by Beckerman et al. [3] in the experimental comparison of the fusion excitation functions of ${ }^{58,64} \mathrm{Ni}+{ }^{58,64} \mathrm{Ni}$ and, explained firstly by Broglia et al. [4] by considering the gained kinematic energy for the (virtual) intermediate states as an important doorway to fusion [5]. Afterwards, many studies $[1,6]$ have been devoted to PQNT effect. But it is still not clarified for the relevant reaction dynamics up to now.

This contribution reports the recent experimental studies for the coupling effect of PQNT on near-barrier fusion with heavy-ions measured by an electrostatic deflector setup. Also a method, based on the CCFULL [7] calculations with suitable inelastic couplings, to sort out the PQNT effect is presented.

\section{Recent near-barrier fusion experiments at CIAE}

The experiments were performed at the HI-13 tandem accelerator of CIAE, Beijing. Fusion evaporation residual (ER) cross sections have been measured by using an electrostatic deflector (see Ref. [8] for more details).

\section{$2.1{ }^{32} \mathbf{S}+{ }^{90,94,96} \mathbf{Z r}$}

The first studied case is ${ }^{32} \mathrm{~S}+{ }^{90,94,96} \mathrm{Zr}[9]$. The idea is to further search for a correlation of sub-barrier fusion enhancement with PQNT channels. 


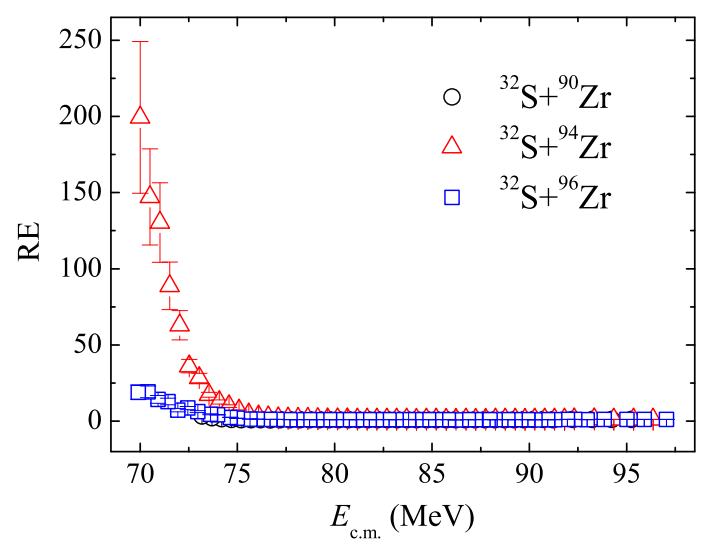

Figure 2: Residual enhancement for near-barrier fusion of ${ }^{32} \mathrm{~S}+{ }^{90,94,96} \mathrm{Zr}$. The original experimental fusion data are taken from Refs. [9].

The fusion excitation functions measured for ${ }^{32} \mathrm{~S}+{ }^{90,94,96} \mathrm{Zr}$ are shown in fig. 1 , where the energy scale $E_{\text {c.m. }}$ (energy in the center-of-mass system) was corrected for the target thickness and the error bars represent purely the statistical uncertainties. It can be seen that the experimental fusion of ${ }^{32} \mathrm{~S}$ $+{ }^{94,96} \mathrm{Zr}$ really shows a strong relation of sub-barrier fusion enhancement to the PQNT channels.

Fusion cross section enhancement reflects the whole effect of couplings to all the relevant channels. For understanding the PQNT effect, the key is to exclude the impact of the collective excitation states. For sorting out the effect related to PQNT, residual enhancement (RE) is defined as the ratio of the experimental fusion data $\left(\sigma_{\operatorname{Exp}}\right)$ to the $\mathrm{CC}$ calculation result $\left(\sigma_{\mathrm{CC}}\right)[7]$ including the major inelastic couplings. That is $\mathrm{RE}=\sigma_{\mathrm{Exp}} / \sigma_{\mathrm{CC}}$, by excluding the inelastic coupling effect considering the good description of the CC theory for the inelastic couplings [2].

The corresponding $\mathrm{RE}$ for ${ }^{32} \mathrm{~S}+{ }^{90,94,96} \mathrm{Zr}$ is shown in fig. 2 . It can be seen that the experimental fusion excitation function of ${ }^{32} \mathrm{~S}+{ }^{90} \mathrm{Zr}$ is reproduced well. While RE shows deviation from unity with the decreasing energy for ${ }^{32} \mathrm{~S}+{ }^{94,96} \mathrm{Zr}$. Notably, bigger RE shows for ${ }^{32} \mathrm{~S}+{ }^{94} \mathrm{Zr}$ than ${ }^{32} \mathrm{~S}+{ }^{96} \mathrm{Zr}$ at the same energy, although of the smaller neutron transfer $Q_{\mathrm{gg}}$-values for the former system and larger neutron separation energies for ${ }^{94} \mathrm{Zr}$. The obtained bigger $\mathrm{RE}$ for ${ }^{32} \mathrm{~S}+{ }^{94} \mathrm{Zr}$ is reasonable considering the similar experimental fusion cross sections for the two systems but the larger inelastic $\left(3^{-}\right)$coupling effect for ${ }^{96} \mathrm{Zr}$. This suggests that more should be considered for a full clarification of the relevant dynamic reaction processes. 


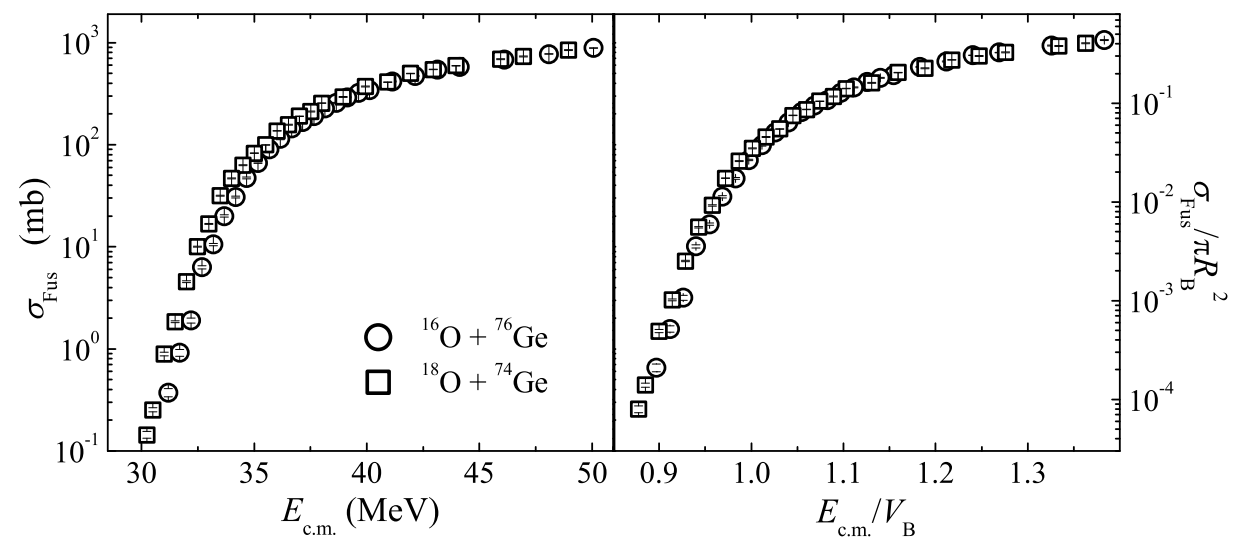

Figure 3: Experimental fusion excitation functions of ${ }^{16} \mathrm{O}+{ }^{76} \mathrm{Ge}$ and ${ }^{18} \mathrm{O}+{ }^{74} \mathrm{Ge}$ (circles and squares, respectively) in absolute (left) and reduced (right) scales.

The role of the neutron orbital populations, such as shell closure of ${ }^{90} \mathrm{Zr}$ and sub-shell closure of ${ }^{96} \mathrm{Zr}$, should be considered theoretically. Certainly, experimental measurements for the transfer reactions of the ${ }^{94,96} \mathrm{Zr}$-involved systems should give a meaningful clue for this quantitative correlation.

\section{$2.2{ }^{16} \mathrm{O}+{ }^{76} \mathrm{Ge}$ and ${ }^{18} \mathrm{O}+{ }^{74} \mathrm{Ge}$}

For simplifying the problem, the systems with only one positive $Q_{-2 \mathrm{n}}$-value neutron transfer channel were selected to study. At first, the fusion of ${ }^{18} \mathrm{O}$ $+{ }^{74} \mathrm{Ge}$ with $Q_{-2 \mathrm{n}}=+3.75 \mathrm{MeV}$ and lower inelastic coupling $\left(Z_{\mathrm{p}} Z_{\mathrm{t}}\right)$ effect was studied experimentally [6].

Fusion of ${ }^{16} \mathrm{O}+{ }^{76} \mathrm{Ge}$ was also measured as a reference. The results of the experimental fusion excitation functions are shown in fig. 3 (left). Usually, a method is used in order to remove the trivial geometric effect for a comparison of the coupling effect between different systems. The right panel shows the reduced fusion excitation functions for the two systems in a reduced energy scale, where $V_{\mathrm{B}}$ is the Coulomb barrier energy and $R_{\mathrm{B}}$ is the fusion barrier radius. One can see that the two reduced fusion excitation functions almost overlap at the whole energy region. This means no visible sub-barrier fusion enhancement related to the positive $Q_{-2 \mathrm{n}}$-value neutron transfer channel for ${ }^{18} \mathrm{O}+{ }^{74} \mathrm{Ge}$ at the measured energy region. This conclusion is consistent with the result that obtained from the experimental studies of ${ }^{36} \mathrm{~S}+{ }^{58} \mathrm{Ni}[10]$ and ${ }^{18} \mathrm{O}+{ }^{A} \mathrm{Sn}$ [11] with positive $Q_{-2 \mathrm{n}}$-value neutron stripping channels. 


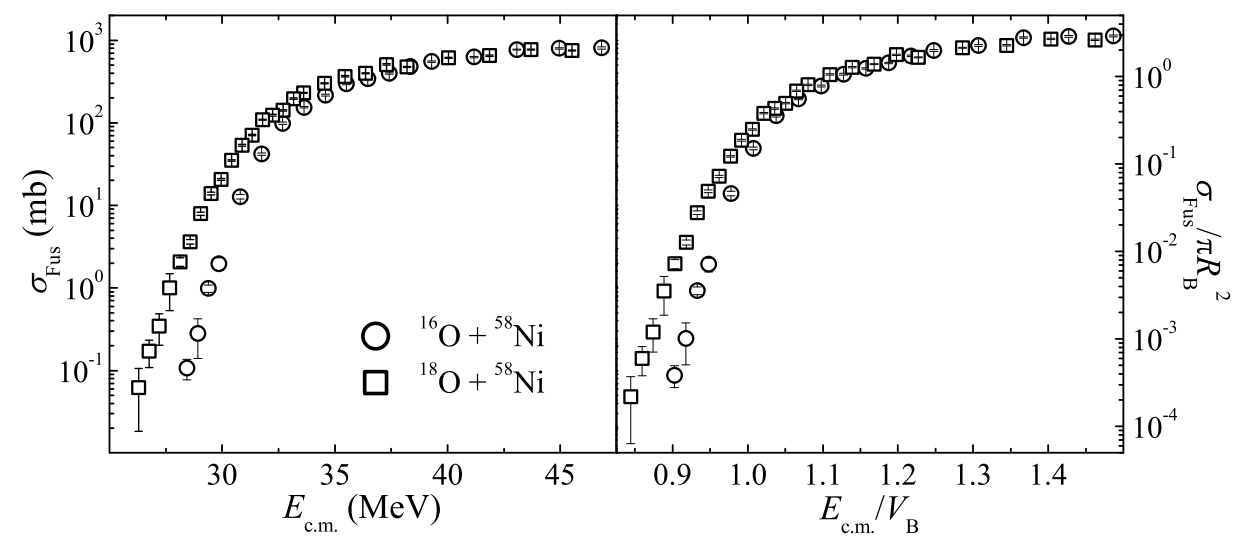

Figure 4: Preliminary experimental fusion excitation functions of ${ }^{16,18} \mathrm{O}+{ }^{58} \mathrm{Ni}$ (circles and squares) in absolute (left) and reduced (right) scales.

\section{$2.3{ }^{16,18} \mathrm{O}+{ }^{58} \mathrm{Ni}$}

For further studying the effect of the neutron transfer channels with positive

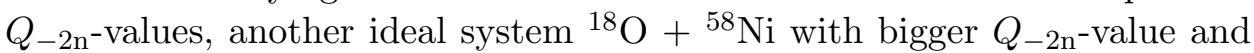
smaller $Z_{\mathrm{p}} Z_{\mathrm{t}}$ was studied. The fusion excitation function of ${ }^{18} \mathrm{O}+{ }^{58} \mathrm{Ni}$ was once measured [12] but only at near-barrier energies and with lower data quality. Fusion of ${ }^{18} \mathrm{O}+{ }^{58} \mathrm{Ni}$ is interesting considering the bigger $Q_{-2 \mathrm{n}} / V_{\mathrm{B}}$ and therefore has attracted many studies, but precise fusion data down to sub-barrier energy region is still absent.

For the moment, we have tried to complete the near-barrier experimental fusion of ${ }^{18} \mathrm{O}+{ }^{58} \mathrm{Ni}$ with rather good accuracy by extending the measurement to the sub-barrier energy region. The fusion of ${ }^{16} \mathrm{O}+{ }^{58} \mathrm{Ni}$ was also measured as a reference system. The Preliminary results of the experimental fusion excitation functions of ${ }^{16,18} \mathrm{O}+{ }^{58} \mathrm{Ni}$ are shown in fig. 4 (left), where the energy scale $E_{\text {c.m. }}$. is corrected for the carbon backing (faced to the beam) and the target thickness. The right panel is what obtained by a representation of the fusion excitation functions in a reduced energy scale. It can be seen that the two reduced fusion excitation functions deviate from each other at near- and sub-barrier energy region. This means the appearance of the PQNT effect on fusion considering the minor coupling effect to the collective inelastic excitation states for the two lighter systems. 


\section{Summary}

The PQNT effect has been studied experimentally. These experimental results further show a complicated PQNT effect, which should be further studied for a full understanding of the underlying physics in the nuclear reactions with heavy-ions.

\section{Acknowledgements}

This work has been supported by the National Key Basic Research Development Program of China under Grant No. 2013CB834404, and the National Natural Science Foundation of China under Grants Nos. 11475263 and 11375268 .

\section{References}

[1] Back B. B. et al., Rev. Mod. Phys., 86 (2014) 317.

[2] Hagino K. and Takigawa N., Prog. Theor. Phys., 128 (2012) 1001.

[3] Beckerman M. et al., Phys. Rev. Lett., 45 (1980) 1472.

[4] Broglia R. A., Dasso C. H., Landowne S., and Winther A., Phys. Rev. C, 27 (1983) R2433.

[5] Henning W. et al., Phys. Rev. Lett., 58 (1987) 318.

[6] Jia H. M. et al., Phys. Rev. C, 86 (2012) 044621.

[7] Hagino K., Rowley N., and Kruppa A. T., Comput. Phys. Commun., 123 (1999) 143.

[8] Zhang Huan-Qiao et al., Chin. Phys. C, 34 (2010) 1628.

[9] Jia H. M. et al., Phys. Rev. C, 89 (2014) 064605.

[10] Stefanini A.M. et al., Nucl. Phys. A, 456 (1986) 509.

[11] Jacobs P. et al., Phys. Lett. B, 175 (1986) 271.

[12] Borges A. M. et al., Phys. Rev. C, 46 (1992) 2360. 